\title{
Критерий сильной локализации на поверхности полупроводника в приближении Томаса-Ферми
}

\author{
(C) В.Б. Бондаренко, А.В. Филимонов \\ Санкт-Петербургский политехнический университет Петра Великого, \\ 195251 Санкт-Петербург, Россия \\ E-mail: enter@spbstu.ru
}

(Получена 21 февраля 2017 г. Принята к печати 28 февраля 2017 г.)

Исследуется характер локализации двумерного электронного газа на поверхности сильно легированного полупроводника, в области пространственного заряда которого проявляется естественный размерный эффект. Из теоретического анализа рассеяния низкоэнергетичных электронов на сформированном на поверхности сосредоточенным зарядом ионизованной примеси хаотическом потенциале определена длина свободного пробега носителя. На основе правила Иоффе-Регеля получен критерий сильной локализации в указанной двумерной электронной системе.

DOI: 10.21883/FTP.2017.10.45015.8507

При анализе размерных отношений в приповерхностном слое легированного полупроводника отмечалось [1], что в большинстве случаев ширина области пространственного заряда (ОПЗ) сравнима со средним расстоянием между примесными атомами - существует естественный размерный эффект. Следовательно, плотность заряда у поверхности не является распределенной и формируется ансамблем дискретных ионизованных атомов. Сосредоточенное распределение примесного заряда создает на поверхности полупроводника хаотический потенциал, который рассеивает электроны в двумерной поверхностной зоне.

Проблема проводимости двумерных электронных систем ранее обсуждалась главным образом применительно к инверсным приповерхностным слоям контактов металл-диэлектрик-полупроводник и квантовым ямам в гетероструктурах [2-15]. Значительное количество экспериментальных данных для этих низкоразмерных систем стимулировало развитие теории; задача становилась двумерной благодаря квантованию движения носителей заряда нормально к плоскости контакта или размерному квантованию в переходных слоях. Очевидно, что наличие электрически активных дефектов у границ раздела по большей части определяет свойства формируемого двумерного электронного газа и особенности токопереноса в указанных структурах. В частности, авторы [2-4] исследовали влияние статистических флуктуаций плотности встроенного заряда в диэлектрике на проводимость инверсных слоев, пренебрегая сильными неоднородностями потенциала на поверхности, вызванными проявлением естественного размерного эффекта в ОПЗ полупроводника.

Рассеяние электронов хаотическим потенциалом приводит к уменьшению длины свободного пробега $l$ уже при температуре абсолютного нуля. В системе любой размерности понятие длины свободного пробега теряет смысл, если ее величина становится меньше длины волны электрона. Тогда электронные состояния локализуются [16]. Согласно правилу Иоффе-Регеля, локализованы состояния, для которых

$$
k l \leq 1
$$

где $k$ - модуль волнового вектора.

Двумерные электронные системы с точки зрения характера их проводимости занимают особое положение в теории конденсированного состояния [17]. Наличие отрицательных и логарифмически расходящихся квантовых поправок к проводимости этих систем, обусловленных волновыми свойствами электрона, исходно приводит к состоянию так называемой слабой локализации $[18,19]$. Поэтому вместо фазового перехода металл-изолятор с ростом беспорядка при выполнении критерия (1) следует говорить о переходе от слабой локализации к сильной [8]. Кроме того, специфические свойства двумерных систем с низкоэнергетичным электронным газом могут усиливать эффекты локализации. В отличие от трехмерных систем, сечение рассеяния медленных электронов [20] растет при уменьшении энергии. Вследствие этого должна сокращаться длина свободного пробега носителя и, возможно, состояние сильной локализации при более низком уровне беспорядка. Поэтому учет естественного размерного эффекта в слоях пространственного заряда, определяющий исходное распределение поверхностного потенциала, является необходимым. Цель настоящей работы заключается в определении условий сильной локализации на поверхности полупроводника при наличии хаотического потенциала, обусловленного случайно распределенными сосредоточенными зарядами в ОПЗ.

Как показали расчеты [1], дискретность распределения заряженных примесей в ОПЗ при макроскопически однородном легировании полупроводника проявляется в широком диапазоне концентраций примеси $10^{16}-10^{20} \mathrm{~cm}^{-3}$, в результате чего неоднородности электрического поля на поверхности растут при увеличении уровня легирования. Наличие поверхностных состояний высокой плотности (порядка $10^{14} \mathrm{~cm}^{-2}{ }_{\ni} \mathrm{B}^{-1}$ и более) позволяет пренебречь пространственной дисперсией диэлектрического 
отклика двумерного электронного газа и определить экранированный поверхностный потенциал в приближении Томаса-Ферми [21]. Однако если уровень Ферми закрепляется у дна поверхностной зоны, то возможна сильная локализация поверхностного заряда вследствие рассеяния низкоэнергетичных носителей. Такая ситуация может реализоваться, например, на поверхности GaP $n$-типа [22].

Для определенности рассмотрим донорный полупроводник при $T=0 \mathrm{~K}$. Имеет смысл говорить о вырожденном полупроводнике, поскольку только в этом случае при нулевой температуре возможно образование обедненного слоя с неэкранированными зарядами примеси. При достаточно больших изгибах зон можно считать, что практически на всей протяженности ОПЗ примесь полностью ионизована.

В приближении Томаса-Ферми потенциальная энергия поверхностного электрона в поле заряженного донора следует за поверхностной плотностью заряда

$$
\sigma_{i}=-\frac{e d_{i}}{4 \pi\left(\rho^{2}+d_{i}^{2} / 4\right)^{3 / 2}} .
$$

Здесь $\rho-$ радиальная координата в плоскости поверхности, $e d_{i}$ - дипольный момент указанного диполя $\left(d_{i}-\right.$ удвоенное расстояние от донора в ОПЗ до плоскости поверхности полупроводника). Основное состояние в соответствующей одноэлектронной задаче формулируется из условия равенства нулю центробежной энергии. Таким образом, волновая функция поверхностного электрона $\psi_{i}(\rho)$ определяется из следующего уравнения Шредингера:

$$
\frac{1}{\rho} \frac{d}{d \rho}\left(\rho \frac{d \psi_{i}}{d \rho}\right)+\frac{2 m}{\hbar^{2}}\left(E-U_{i}\right) \psi_{i}=0 .
$$

Конечное нормированное решение (3), удовлетворяющее условию $\sigma_{i}=-e\left|\psi_{i}\right|^{2}$ и имеющее вид

$$
\psi_{i}(\rho)=\sqrt{\frac{d_{i}}{4 \pi}}\left(\rho^{2}+\frac{d_{i}^{2}}{4}\right)^{-3 / 4},
$$

определяет при нулевом собственном значении потенциальную энергию поверхностного электрона в самосогласованном поле:

$$
U_{i}(\rho)=-\frac{3\left(d_{i}^{2}-3 \rho^{2}\right)}{8 \pi D_{0}\left(\rho^{2}+d_{i}^{2} / 4\right)^{2}} .
$$

Здесь $D_{0}=m / \pi \hbar^{2}-$ локальная плотность электронных состояний, $m$ - эффективная масса электрона в поверхностной зоне. Рассмотрим рассеяние исходно свободного носителя на указанном потенциале. Для этого прежде всего необходимо учесть наличие на поверхности хаотического потенциала с характерной амплитудой [1]

$$
\delta U=\frac{N_{0}}{D_{0} \sqrt{\pi N_{S}}},
$$

где $N_{0}-$ уровень легирования полупроводника, $N_{S}=N_{0} L_{0}-$ поверхностная концентрация заряда, $L_{0}-$ ширина области обеднения. Имея конкретный вид закона распределения потенциальной энергии поверхностного электрона, получаем соответствующую плотность состояний. Согласно приведенному в [23] методу, исходное выражение имеет вид

$$
D(E)=\int_{-\infty}^{E} D_{0}(E-U) f(U) d U,
$$

где $D_{0}(E)$ - невозмущенная плотность электронных состояний, $f(U)$ - функция плотности распределения потенциальной энергии электрона $U$ на поверхности; энергия поверхностных носителей $E$ при этом отсчитывается от дна невозмущенной поверхностной зоны. При параболическом законе дисперсии плотность поверхностных состояний в отсутствии хаотического потенциала является константой. Поэтому (7) можно переписать:

$$
D(E)=D_{0} \int_{-\infty}^{E} f(U) d U
$$

Конкретный вид функциональной зависимости $D=D(E)$ таким образом определяется характером распределения хаотического потенциала. Некоррелированное расположение кулоновских центров - заряженной примеси в ОПЗ полупроводника - предполагает их пуассоновское распределение по количеству частиц. При условии линейности и локальности экранирования в двумерной электронной системе и учете принципа суперпозиции полей вполне адекватна модель гауссового распределения хаотического потенциала [24]. В силу этого можно принять соответствующее распределение потенциальной энергии электрона в поверхностной зоне:

$$
f(U)=\frac{1}{\delta U \sqrt{2 \pi}} \exp \left(-\frac{U^{2}}{2 \delta U^{2}}\right) .
$$

Вычисление интеграла в (8) с указанным видом функции плотности распределения (9) приводит к известному из теории вероятности результату через функцию ошибок:

$$
D(E)=\frac{D_{0}}{2}\left[1+\operatorname{erf}\left(\frac{E}{\delta U \sqrt{2}}\right)\right] .
$$

При известной поверхностной плотности заряда последнее выражение позволяет получить уравнение для энергии Ферми:

$$
N_{S}=\int_{-\infty}^{E_{\mathrm{F}}} D(E) d E
$$

Далее в пределе низких энергий поверхностных носителей можно с хорошей точностью заменить сложную функциональную зависимость (11) на линейную аппроксимацию:

$$
N_{S} \approx D_{0}\left(\frac{\delta U}{\sqrt{2 \pi}}+\frac{E_{\mathrm{F}}}{2}\right)
$$


С учетом (6) получаем, что $N_{S}(0)=N_{0}^{2 / 3} /\left(2 \pi^{2}\right)^{1 / 3}$. Таким образом, величина волнового вектора электрона на уровне Ферми определяется выражением

$$
k_{\mathrm{F}}=\sqrt{4 \pi\left[N_{S}-\frac{N_{0}^{2 / 3}}{\left(2 \pi^{2}\right)^{1 / 3}}\right]} .
$$

Для медленных электронов сечение рассеяния не зависит от угла и ограничивает длину свободного пробега электрона в поверхностной зоне:

$$
l=\frac{1}{s N_{S}} .
$$

В данном случае $s$ - среднее сечение рассеяния на флуктуациях потенциала. Сечение рассеяния медленных электронов в двумерном случае на некотором локальном потенциале можно записать в виде [20]

$$
s_{i}=\frac{\pi^{2}}{k_{\mathrm{F}}}\left(\ln ^{2} \frac{2}{\gamma k_{\mathrm{F}} r_{i}}+\frac{\pi^{2}}{4}\right)^{-1}
$$

где $\gamma=\exp (C), C \approx 0.577 \ldots-$ постоянная Эйлера, $r_{i}$ - величина с размерностью длины. Определение $r_{i}$ производится в процессе сшивки волновой функции электрона $\psi_{i}$ в областях $\rho \leq \rho_{i}$ и $\rho_{i}<\rho<\frac{1}{k_{F}}$. Во внутренней области уравнение Шредингера имеет вид

$$
\frac{1}{\rho} \frac{d}{d \rho}\left(\rho \frac{d \psi_{i}}{d \rho}\right)-\frac{2 m}{\hbar^{2}} U_{i}(\rho) \psi_{i}=0 \text {, }
$$

где потенциальная энергия $U_{i}$ должна соответствовать распределению поверхностного заряда (2). Вдали от рассеивающего центра потенциальная энергия электрона становится малой. При этом решение уравнения (16) приводит к выражению

$$
\psi_{i} \approx C_{1}+C_{2} \ln \rho .
$$

Сшивая логарифмическую производную волновой функции на границе $\rho=\rho_{i}$ и учитывая поведение волновой функции (4) при $\rho \gg d_{i}$, имеем

$$
\frac{C_{1}}{C_{2}}=-\ln \left[\rho_{1} \exp \left(\frac{2}{3}\right)\right] .
$$

Выражение с размерностью длины, стоящее в (18) под логарифмом, и есть $r_{i}$. Значение $\rho_{i}$ определяется из условия $U_{i}(\rho)=0$. Таким образом,

$$
r_{i}=\frac{d_{i}}{\sqrt{3}} \exp \left(\frac{2}{3}\right)
$$

Теперь для вычисления $s$ необходимо усреднить выражение (15) по всем размерам диполей $d_{i}$, равномерно распределенных в диапазоне $0-2 L_{0}$ :

$$
s=\frac{\pi^{2}}{2 k_{\mathrm{F}}^{2} L_{0}} \int_{0}^{2 k_{\mathrm{F}} L_{0}}\left[\ln ^{2}\left(\frac{2 \sqrt{3}}{\eta \xi}\right)+\frac{\pi^{2}}{4}\right]^{-1} d \xi .
$$

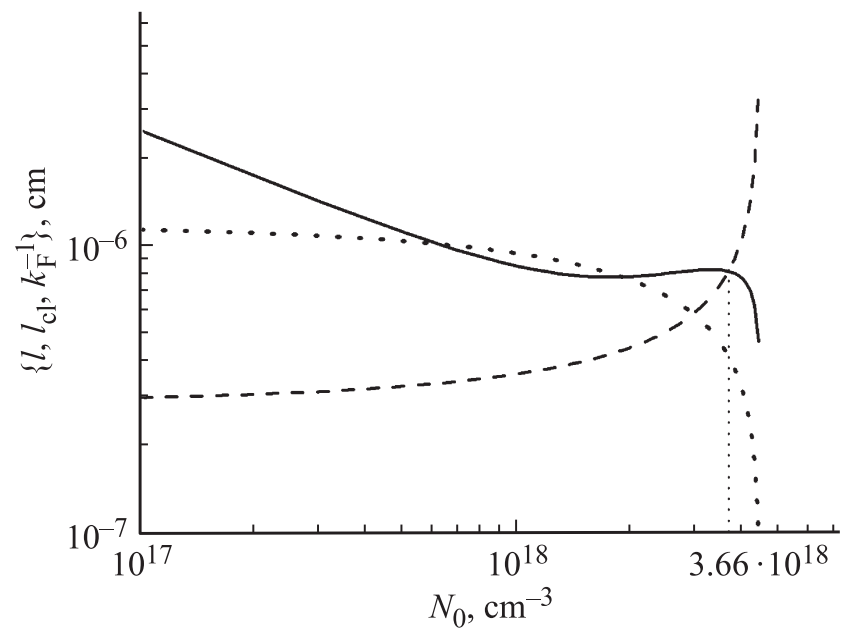

Зависимости длины свободного пробега поверхностного электрона (сплошная кривая) и обратной величины волнового вектора носителя (штриховая кривая) от уровня легирования при значении поверхностной концентрации заряда $N_{S}=10^{12} \mathrm{~cm}^{-3}$. Указано критическое (при условии $k_{\mathrm{F}} l=1$ ) значение уровня легирования $N_{0}=3.66 \cdot 10^{18} \mathrm{~cm}^{-3}$. Для заданной концентрации $N_{S}$ приведена зависимость $l_{c 1}=l_{c 1}\left(N_{0}\right)$ (пунктирная кривая).

Здесь введено обозначение $\eta=\exp (2 C / 3)$. Интеграл в выражении (20) подстановкой $t=\ln (2 \sqrt{3} / \eta \xi)$ приводится к виду

$$
I(y)=\frac{2 \sqrt{3}}{\eta} \int_{y}^{\infty} \frac{\exp (-t)}{t^{2}+\pi^{2} / 4} d t
$$

где $y=\ln \left(\sqrt{3} / \eta k_{\mathrm{F}} L_{0}\right)$. Последняя функциональная зависимость (21) может быть представлена суперпозицией интегральных показательных функций комплексного аргумента [25]:

$$
I(y)=\frac{2 \sqrt{3}}{\pi \eta}\left[E i\left(-y+\frac{i \pi}{2}\right)+E i\left(-y-\frac{i \pi}{2}\right)\right] .
$$

Рассчитанная по формулам (14), (20)-(22) зависимость длины свободного пробега носителя на уровне Ферми от концентрации $N_{0}$ при фиксированном значении $N_{S}$, представлена графически на рисунке. Для сравнения на указанном графике приведена классическая длина свободного пробега электрона $l_{c 1}$, которая может быть установлена из определения в качестве сечения рассеяния размера области, классически недоступной для делокализованного носителя, т.е. из условия $E_{\mathrm{F}}=U_{i}(\rho)$. В данном случае значение сечения рассеяния медленных электронов оценивается из асимптотического выражения потенциальной энергии (5) для аргументов $\rho>d_{i}$. Имея в виду зависимость $E_{\mathrm{F}}$ от уровня легирования из выражения (13), получаем, что

$$
l_{c 1} \approx \frac{2}{3 N_{S}} \sqrt{\pi\left[N_{S}-\frac{N_{0}^{2 / 3}}{\left(2 \pi^{2}\right)^{1 / 3}}\right]} .
$$


Для идентификации состояния системы вблизи значения $E_{\mathrm{F}}=0$ (или $\left.k_{\mathrm{F}}=0\right)$ потребуется асимптотика $I(y)$ при $y \gg 1$. С учетом поведения (22) в области больших аргументов для среднего сечения рассеяния имеем

$$
s \approx \frac{\pi^{2}}{k_{\mathrm{F}}} \ln ^{-2}\left(\frac{\sqrt{3}}{\eta k_{\mathrm{F}} L_{0}}\right) .
$$

Теперь возможна оценка состояния сильной локализации на поверхности полупроводника. Следуя (14) и учитывая выражение для ширины ОПЗ $L_{0}=N_{S} / N_{0}$, на основе правила (1) получаем критерий

$$
\frac{k_{\mathrm{F}}^{2}}{\pi^{2} N_{S}} \ln ^{2}\left(\frac{\sqrt{3} N_{0}}{\eta k_{\mathrm{F}} N_{S}}\right) \leq 1 .
$$

Проведем анализ полученных результатов. Прежде всего следует отметить некоторую немонотонность зависимости длины свободного пробега поверхностного электрона от уровня легирования полупроводника (см. рисунок). Данное изменение характера зависимости при фиксированной плотности поверхностного заряда (т.е. при постоянном количестве центров рассеивания на единице поверхности) связано с уменьшением характерного масштаба изменения рассеивающего потенциала (5) при общем сокращении ширины ОПЗ $L_{0}$. Из критерия (25) следует более низкий уровень беспорядка (меньшее значение $N_{0}$ ) в системе, при котором реализуется состояние сильной локализации, по сравнению с классическим перколяционным порогом [23]. Согласно решению известной континуальной задачи теории протекания в двумерной системе с хаотическим потенциалом, статистические свойства которого симметричны относительно значения $U=0$, порог протекания равен нулю. В рассматриваемой электронной системе с гауссовым беспорядком это соответствует условию $E_{\mathrm{F}}=0$ (или $\left.l_{c 1}=0\right)$. Сравнение указанного перколяционного порога c рассчитанным показывает, что критическая концентрация кулоновских центров $N_{0}$ оказывается несколько меньше. Кроме того, значение поверхностной плотности $N_{S}$, отвечающее критерию сильной локализации, близко к типичным величинам, характерным для поверхности легированных полупроводников с диэлектрической проницаемостью порядка 10. Таким образом, для указанных систем в большинстве случаев двумерный электронный газ уже может находиться в состоянии сильной локализации, если уровень Ферми закрепляется у дна поверхностной зоны.

Результаты были получены в СПбПУ в рамках выполнения государственного задания Минобрнауки России.

\section{Список литературы}

[1] В.Б. Бондаренко, М.В. Кузьмин, В.В. Кораблев. ФТП, 35, 964 (2001)

[2] В.А. Гергель, Р.А. Сурис. ЖЭТФ, 75, 191 (1978).

[3] В.А. Гергель, Р.А. Сурис. ЖЭТФ, 84, 719 (1983).
[4] В.А. Гергель, Г.В. Шпатаковская. ФТП, 27, 923 (1993).

[5] S.V. Kravchenko, W.E. Mason, G.E. Bowker, J.E. Furnaux, V.M. Pudalov, M.D. Iorio. Phys. Rev. B, 51, 7038 (1995).

[6] F.W. Van Keuls, H. Mathur, H.W. Jiang, A.J. Dahm. Phys. Rev. B, 56, 13263 (1997).

[7] A. Koukitu, Y. Kumagai, H. Seki. Phys. Status Solidi A, 180, 115 (2000).

[8] А.А. Шерстобитов, Г.М. Миньков, О.Э. Рут, А.В. Германенко, Б.Н. Звонков, Е.А. Ускова, А.А. Бирюков. ФТП, 37, 730 (2003).

[9] Н.В. Агринская, В.И. Козуб, Д.В. Полоскин, А.В. Черняев, Д.В. Шамшур. Письма ЖЭТФ, 80, 36 (2004).

[10] M. Gonschorek, J.F. Carlin, E. Feltin, M.A.Py.N. Grandjean. Appl. Phys. Lett., 89, 062106 (2006).

[11] R. Tulek, A. Ilgaz, S. Gokden, A. Teke, M.K. Ozturk, M. Kasap, S. Ozcelik, E. Arsalan, E. Ozbay. J. Appl. Phys., 105, 013707 (2009).

[12] J. Xue, J. Zhang, W. Zhang, L. Li, F. Meng, M. Lu, J. Ning, Y. Hao. J. Cryst. Growth, 343, 110 (2012).

[13] К.П. Калинин, С.С. Криштопенко, К.В. Маремьянин, К.Е. Спирин, В.И. Гавриленко, А.А. Бирюков, Н.В. Байдусь, Б.Н. Звонков. ФТП, 47, 1497 (2013).

[14] А.В. Германенко, Г.М. Миньков, О.Э. Рут, А.А. Шерстобитов, С.А. Дворецкий, Н.Н. Михайлов. ФТП, 49, 41 (2015).

[15] И.Л. Калентьева, О.В. Вихрова, А.В. Здоровейщев, Ю.А. Данилов, А.В. Кудрин. ФТП, 50, 204 (2016).

[16] A.F. Ioffe, A.R. Regel. Prog. Semicond., 4, 237 (1960).

[17] В.Ф. Гантмахер. Электроны в неупорядоченных средах (М., Физматлит, 2003).

[18] M. Imada, A. Fujimori, Y. Tokura. Rev. Mod. Phys., 70, 1039 (1998).

[19] F. Evers, A.D. Mirlin. Rev. Mod. Phys., 80, 1355 (2008).

[20] Л.Д. Ландау, Е.М. Лифшиц. Теоретическая физика: 3. Квантовая механика (нерелятивистская теория) (М., Наука, 1989).

[21] В.Б. Бондаренко, В.В. Кораблев, Ю.И. Равич. ФТП, 38, 331 (2004).

[22] J. Wagner, W. Stolz, K. Ploog. Phys.Rev., B, 32, 4214 (1985).

[23] Б.И. Шкловский, А.Л. Эфрос. Электронные свойства легированных полупроводников (М., Наука, 1979).

[24] В.Л. Бонч-Бруевич, И.П. Звягин, Р. Кайпер, А.Г. Миронов, Р. Эндерлайн, Б. Эссер. Электронная теория неупорядоченных полупроводников (М., Наука, 1981).

[25] Н.Н. Лебедев. Специальные функции и их приложения (М., Гостехтеоретиздат, 1953).

Редактор Г.А. Оганесян

\section{A criterion for strong localization on a semiconductor surface in the Thomas-Fermi approximation}

\section{V.B. Bondarenko, A.V. Filimonov}

\section{Peter the Great St. Petersburg Polytechnic University, 195251 St. Petersburg, Russia}

\begin{abstract}
We study the localization of the two-dimensional electron gas on the surface of the highly doped semiconductor, in the space charge region which manifests a natural size effect. From the theoretical analysis of the scattering of low-energy electrons on the surface formed on focused charged ionized impurity chaotic potential defined mean free path of the carrier. On the basis of the Ioffe-Regel criterion is entitled to receive a strong localization in this two-dimensional electron system.
\end{abstract}

